\title{
THE DANGER OF INCORRECT EXPECTATIONS IN DRIVING: THE FAILURE TO RESPOND
}

\author{
Marieke H. Martens \\ TNO Human Factors \\ Soesterberg, the Netherlands \\ E-mail: marieke.martens@tno.nl
}

\begin{abstract}
Summary: This paper addresses Inattentional Blindness or look-but-fail-to-see in driving. How it is possible that visual information, highly relevant for driving is not perceived or responded to. Our main focus was on driver expectations. Different experimental studies were performed. In the conducted studies, we had drivers perform a driving task in which they had to drive a specific road numerous times. This way they were able to control driver expectations with a specific road environment. After some drives we made some crucial changes to the road environment (e.g., change in priority, change in No-Entry road). Behavioural responses (speed, deceleration) were measured to these changes, as well as eye movenents (glance duration) and awareness of the changes. All partcipants were experienced drivers, and over all studies, 250 drivers participated. There was a relation between driver expectations and the failure to respond. Glance duration to traffic signs was found to decrease with a driver becoming more and more familiar with a specific road. Visually selecting the information seems to be an important condition for enabling a response, but it is certainly not enough. There are various cases where expectations were so strong that drivers looked at the information but did not respond. There seems to be a high correlation between glance duration and responding, as well as between the type of change and responding. In-vehicle equipment warning the driver for these types of situations is highly effective. An elaborated task performance model is presented.
\end{abstract}

\section{INTRODUCTION}

When a road user drives on a road, different aspects of the environment demand and compete for attention. The question we want to address is: How does this process of allocating attention to different objects work, and how do drivers select information? In an ideal world, the items that are relevant for the task at hand are selected for further processing and are processed to a deeper extent. However, reality is shown to be less ideal.

This paper focuses on the effect of expectations on drivers' visual scanning behaviour and the way drivers process visual information in the outside world. It discusses the results found in several experimental studies and presents an elaborated task performance model, based on Rasmussen's model (1986).

\section{EFFECT OF EXPECTATIONS IN NON-DRIVING SITUATIONS}

In three different laboratory experiments (Martens, 2004; Martens \& Slegers, 2002) participants were presented with a dynamic and abstract environment on a computer monitor. We 
investigated the effects of expectations on eye glance duration and on the failure to respond to relevant information. Glance duration and manual responses to predefined targets amongst distractors were measured in a dynamic and abstract environment shown on a computer monitor. Participants received a continuous flow of stimuli with either a predictable sequence of targets and distractors or with a random sequence. In the predictable sequence, participants were able to develop expectations about the order of targets and distractors.

\section{Glance Duration}

What we found is that under those conditions, glance duration for targets was longer than for distractors only for the predictable condition $[\mathrm{F}(1,21)=16.65, \mathrm{p}<0.0005]$.

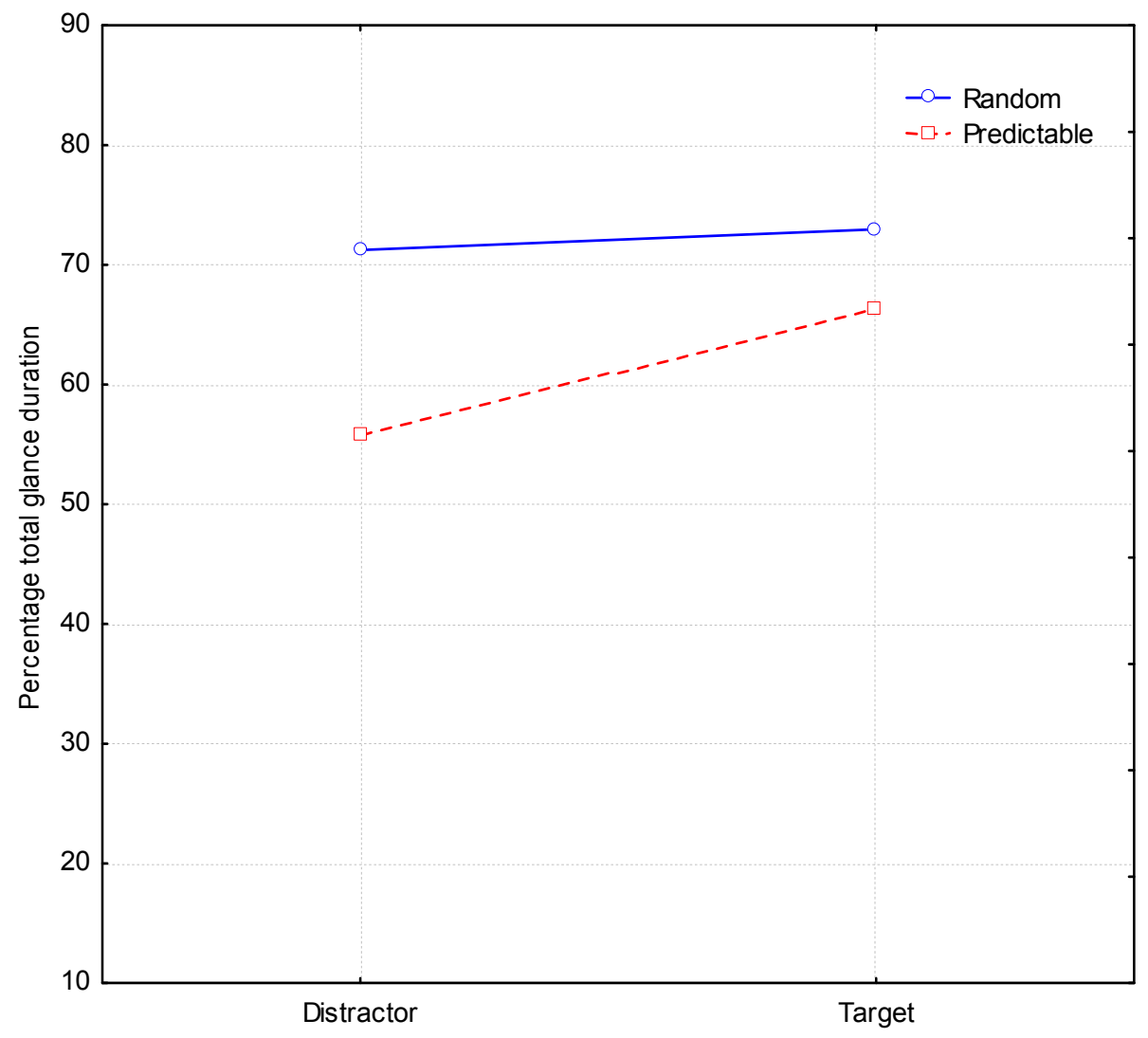

Figure 1. The interaction effect between condition (random and predictable) and stimulus (target and distractor) on percentage glance duration in Session 1 of experiment 1, with shorter glances at the distractors in the predictable condition.

\section{Response}

Also, responses to unexpectedly presented targets were much slower than the mean response time to targets $[\mathrm{t}(4)=4.15, \mathrm{p}<0.014]$. 


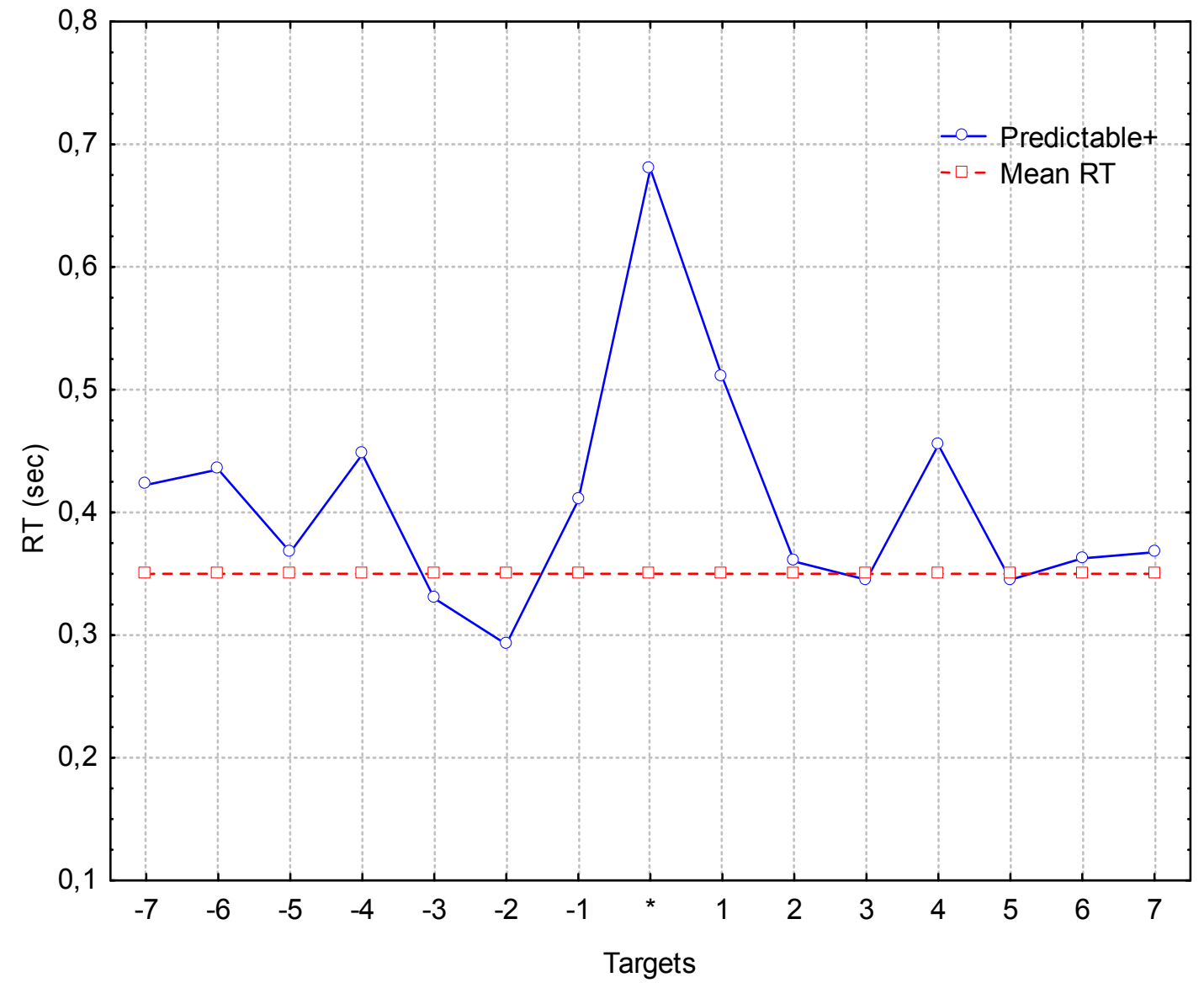

Figure 2. The RTs of the predictable condition around the time of the irregularity, indicated by *, in comparison to the mean $R T$ in that condition

\section{EFFECT OF EXPECTATIONS IN DRIVING SITUATIONS}

For the driving situation, these results may mean that if drivers get familiar with a certain type of road, they spend less time looking at the traffic signs and signals.

In order to see if this was the case, we performed a driving study with 28 paid participants (Martens \& Fox, 2007). Participants were randomly assigned to one of two experimental conditions; the field condition (real driving) or the video condition (watching the same road on video). All participants were experienced drivers.

The route was a two-lane rural road (speed limit $80 \mathrm{~km} / \mathrm{h})$, with a small urban area $(50 \mathrm{~km} / \mathrm{h})$. The total length of the route was 18 kilometers. Participants in the field condition were required to drive from the starting point to an end point, make a U-turn and drive back to the starting point. This was repeated four times in a row per day on three different days (total of 12 drives). 


\section{Glance Duration}

For total glance duration, there was a main effect of Day $[\mathrm{F}(2,52)=47.23 ; \mathrm{p}<0.0001]$, with a decrease in glance duration over days. In general, it can be claimed that the more often people encounter the same road environment, the less time they spend glancing at objects. There was an interaction between Day and Condition $[F(2,52)=3.84 ; \mathrm{p}<0.028]$, which is shown in Figure 3 .

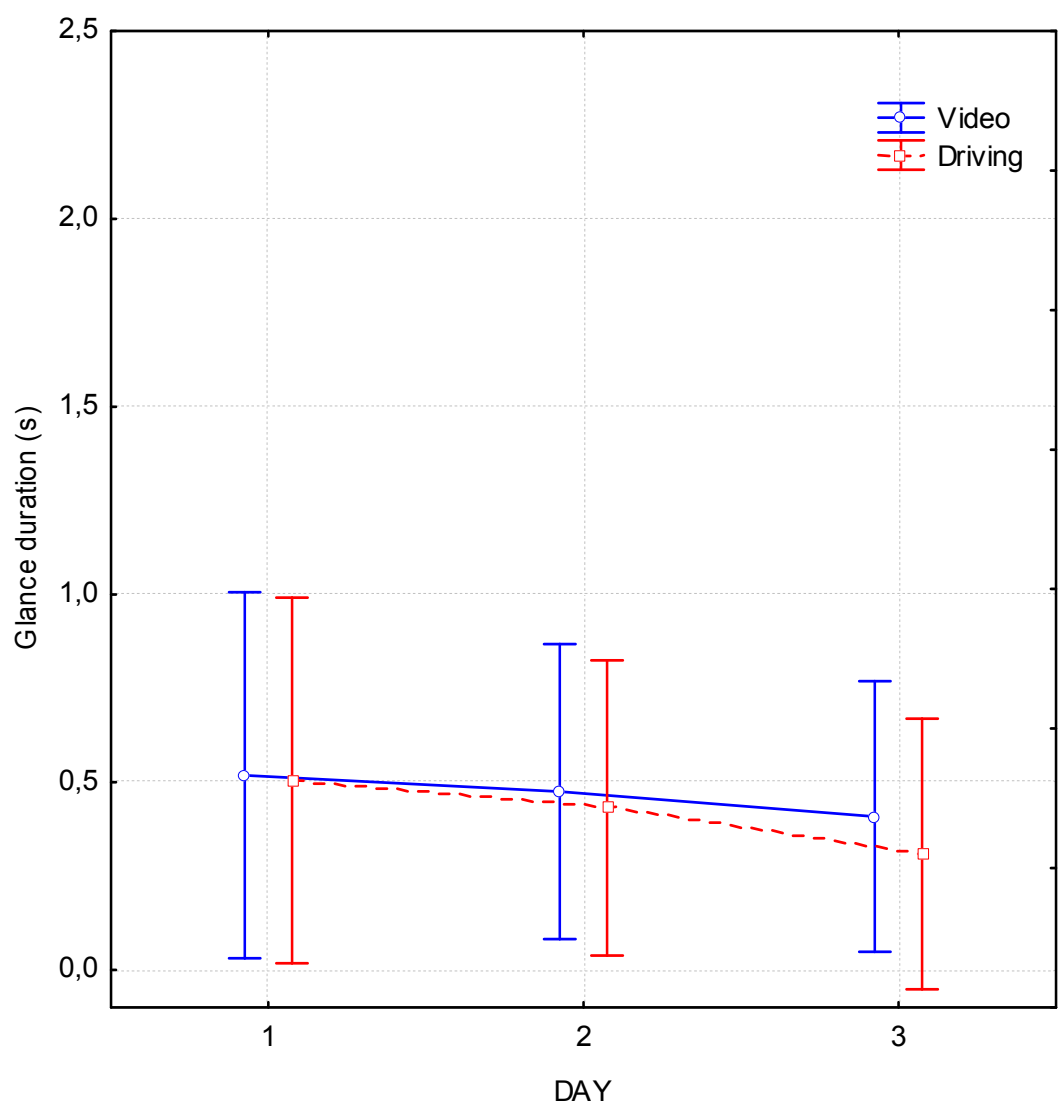

Figure 3. The 2-way interaction between Condition and Day on total glance duration(s) (The error bars indicate the standard error)

\section{Response}

The next question is whether this reduced glance duration to road signs with increasing familiarity with the road also results in a driver failure to adequately respond to information. In a low-cost driving simulator and a high-end driving simulator, these issues were studied.

The low-cost driving simulator study investigated the effect of expectations on glance duration and on responding to unexpected information (Martens \& Fox, in press). Participants drove a low-cost simulator while their eye movements were recorded. After driving the same rural route and one urban area 24 times ( 5 drives per day), participants were confronted with a change-inpriority situation. In the first drives they had priority, and on their last drive there were priority road markings on the road and a different traffic sign, indicating that they should give priority. 
Again, the study showed that with repeated exposure, participants spent less time glancing at traffic signs along the route. When the priority situation was changed without any specific instruction, drivers did glance at the sign indicating the new situation but did not process the information sufficiently to respond adequately. Repeated exposure to the same road resulted in many inadequate responses to the change in priority, even though there was a new sign and there were priority road markings that were not present before.

The high-end driving simulator study addressed the central question of whether the failure to adequately respond due to familiarity with the road mainly occurs if drivers drive the same road several times or whether it also occurs if the road environment partly varies from one drive to the other (Martens, 2005). In order to study this, a normal road was changed into a No-Entry road after 19 drives. Participants were warned about this change in various ways, from just putting up the No-Entry sign to additional signs warning of the change via auditory in-vehicle messages. The driving simulator study showed that there was no difference in the inadequacy of the response between the same drives and the varied drives. Even more, the failure to adequately respond (do not enter the road) was not even the negative consequence of driving the same or a similar road numerous times, but rather the result of expectations that the drivers already had on the first drive. This indicates that there are situations in which the failure to respond is not the mere result of prior exposure to a particular road, but rather the result of the wrong expectations induced by the road design itself. Even on the first drive, one did not expect the No-Entry situation, leading to drivers entering this road. Although fewer errors were made in the case of an additional yellow sign indicating a change in the traffic situation, there were still people who entered the road. Only by means of an active auditory in-vehicle message, were there no participants that entered the No-Entry road.

\section{DRIVER TASK PERFORMANCE MODEL}

If we want to model this type of phenomenon, a solid basis is the task performance model of Rasmussen (1986). The driving task is often analysed in terms of Rasmussen's three performance levels. The three levels of behaviour that Rasmussen distinguishes are the knowledge-based, rule-based and skill-based level.

We developed a new model, which is the elaborated task model, presented in Figure 4. According to the elaborated task model, the difference between the three levels of performance is explained by the level of bottom-up and top-down control, the level of practice, the level of attention and time that a task requires, the level of arousal, and the level of task load. At each level, the risk for the failure to respond to relevant information results from a different cause.

In case of knowledge-based performance (new type tasks, for instance driving in a city centre for the first time), there is a high task load, but if the task load does not exceed the available attentional resources, performance may still be fairly good. Because the task is new and is hardly practiced, performing it takes quite some time, arousal is relatively high and selection and processing basically occurs in a bottom-up manner. In this case 'the failure to apprehend' is a result of failure to select the right information at the right time because of a limited processing capacity. 


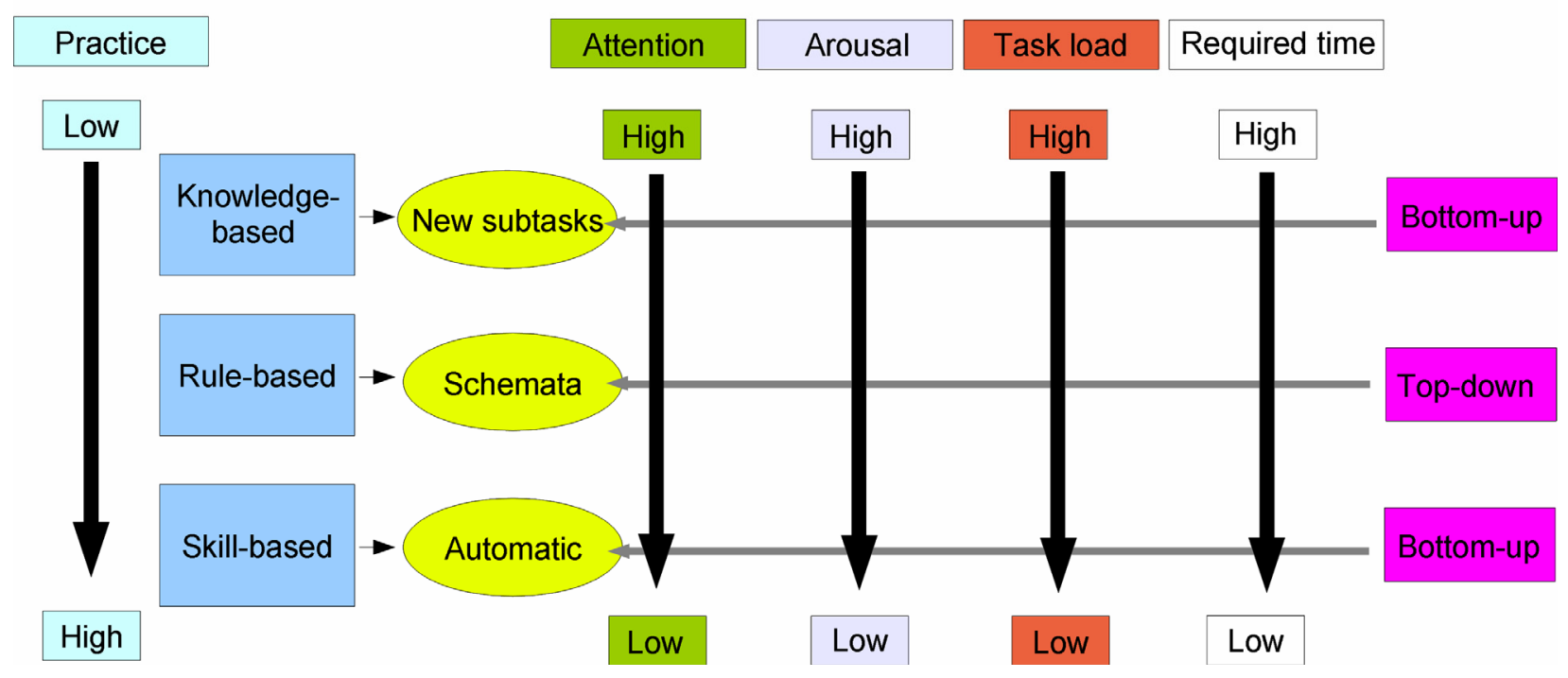

Figure 4. The elaborated task performance model, based on Rasmussen (1986)

In case of rule-based performance (if... then... type of situations, such as giving priority), drivers have a strong top-down control and depend on the activated schema to select and process information. The task is fairly well practised, the level of arousal is not so high, nor is attention, task load or the time required for performing the task. 'The failure to apprehend' is the result of the strong top-down control, which is so strong that it does not allow the bottom-up input of signals that do not fit the top-down schemata.

Finally, in the case of skill-based behaviour or automatic task performance (for example lane keeping), there is a direct link between a stimulus and a response. Information processing and responding is carried out entirely bottom-up, without any room for top-down control. The task is highly practised and the required level of attention is low; these tasks are not very demanding, arousal is normally very low and the time needed for performing the task is minimal. Therefore, in skill-based behaviour the failure to respond to relevant information is explained by the lack of top-down control.

\section{REFERENCES}

Martens, M.H. (2004). Stimuli fixation and manual response as a function of expectancies. Human Factors, 46(3), 410-423.

Martens, M.H. (2005). Responding to Changes in the Traffic Situation: Does experience make a difference? TNO Report TNO-DV3 2005 D018. Soesterberg, the Netherlands.

Martens, M.H. \& Fox, M.R.J. (In press). Do Familiarity and Expectations Change Perception? Drivers' Glances and Response to Changes. Transportation Research Part F.

Martens, M.H., \& Fox, M. (2007). Does road familiarity change eye fixations? A comparison between watching a video and real driving. Transportation Research Part F, 10(1), 33-47. 
Martens, M.H., \& Slegers, K. (2002). The role of expectations and implicit conjunctions on target detection and eye movements. TNO Report TM-02-D007. Soesterberg, the Netherlands. 